\title{
Die „subjektive Seite“ von Zwang und Gewalt in der Psychiatrie
}

\author{
The „Subjective Aspects“ of Restraint and Violence in Psychiatry
}

Autoren

Institute
Ullrich Meise' ${ }^{1}$, Beatrice Frajo-Apor ${ }^{2}$

Gesellschaft für Psychische Gesundheit - pro mente tirol, Innsbruck/Österreich

2 Department für Psychiatrie und Psychotherapie, Medizinische Universität Innsbruck/Österreich

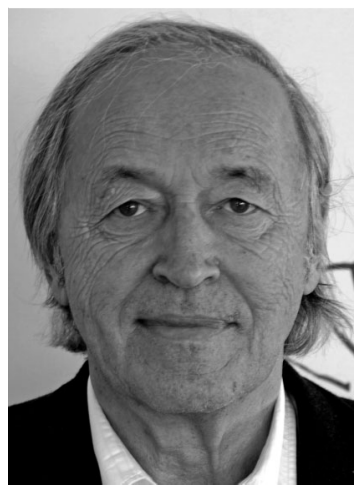

Univ.-Prof. Dr. Ullrich Meise

\section{Bibliografie}

DOI http://dx.doi.org/

10.1055/s-0030-1266104

Psychiat Prax 2011;

38: 161-162

(c) Georg Thieme Verlag KG

Stuttgart · New York .

ISSN 0303-4259

\section{Korrespondenzadresse}

\section{Univ.-Prof. Dr. Ullrich Meise}

Gesellschaft für Psychische

Gesundheit - pro mente tirol

Karl-Schönherr-Straße 3

6020 Innsbruck, Österreich

meise.u@gpg-tirol.at
Die Anwendung von Zwangsmaßnahmen - Unterbringung, Fixierung, Isolierung und Zwangsbehandlung - aber auch von informellem Zwang [1] sind weltweit ein charakteristischer Teil der psychiatrischen Versorgung. Sie gehören zur Schattenseite der psychiatrischen Tätigkeit und die Diskussion darüber ist so alt wie die Psychiatrie selbst [2]. Das Dilemma der Psychiatrie liegt in dem doppelten Mandat, sie hat sowohl eine therapeutische Aufgabe als auch eine staatliche Ordnungsfunktion. Die Möglichkeit, Menschen mit psychischen Störungen wider deren Willen zu behandeln und somit - zumindest vorübergehend ihre Autonomie und Eigenverantwortung durch Fremdbestimmung und Fürsorge zu ersetzen, erfordert folgende Voraussetzungen:

- den Verlust der Einsichts- und Urteilsfähigkeit und folglich die teilweise oder völlige Aufhebung der Freiheit zur autonomen Willensbildung und

- eine im Gefolge der psychischen Erkrankung konkrete Selbst- oder Fremdgefährdung.

Der durch Freiheitsbeschränkung verursachte Eingriff in die individuellen Persönlichkeitsrechte, wobei im Falle einer Zwangsbehandlung auch die Unversehrtheit der Person betroffen ist, gründet somit auf 2 Voraussetzungen: Dem „Fürsorgegedanken“ und dem Gedanken der „Gefahrenabwehr“. Auf diesen Grundsätzen fußen jene gesetzlichen Bestimmungen in den einzelnen EU-Ländern, die diese Maßnahmen sanktionieren. Trotzdem sind diese Gesetze verschieden, und die Entscheidung, Zwangsmaßnahmen anzuwenden, wird unterschiedlich gehandhabt. Daher finden sich zwischen den einzelnen Staaten, was die Art, Dauer und Häufigkeit von Zwangsmaßnahmen betrifft, erhebliche Differenzen [3-5]. Verantwortlich dafür sind u.a. auch der fehlende Konsens, welche Maßnahmen als Zwang registriert werden, sowie unterschiedliche Definitionen [6]. Es wird von der Europäischen Kommission und dem Europarat als notwendig erachtet, innerhalb der Union in dieser Angelegenheit eine Harmonisierung herbeizuführen $[7,8]$.

Insgesamt liegt der durch die Psychiatrie ausgeübte Zwang nicht im Fokus der klinischen Forschung [9]. Das Wissen über das Ausmaß der Anwendung von freiheitsbeschränkenden Maßnahmen ist lückenhaft. Die für einzelne Länder verfügbaren Daten sind untereinander schwer vergleichbar, es finden sich aber auch große Abweichungen zwischen den Kliniken innerhalb einzelner Länder. Dadurch kann der Eindruck entstehen, dass das Ausmaß und die Art von Zwangsmaßnahmen stärker von Kontextfaktoren und weniger von der Psychopathologie der Patienten abhängen. Neben der Erfassung von administrativen oder deskriptiven Aspekten wie der Art, Häufigkeit oder der Dauer dieser Einschränkungen sind noch viele Fragen offen, zu denen bislang kaum Antworten vorliegen. Dazu einige Gesichtspunkte, die etwas Licht auf die im Titel angesprochene „subjektive Seite“ werfen könnten:

1. Welchen Einfluss haben Zwangserfahrungen auf das Erleben und den Selbstwert von Patienten?

2. Beeinflussen solche Gewalterfahrungen den weiteren Krankheitsverlauf, eine zukünftige Inanspruchnahme psychiatrischer Leistungen oder die Compliance?

3. Begünstigen Zwangsmaßnahmen das Fortbestehen oder die Entwicklung einer PTSD?

4. Werden die Anwendung von Zwang oder Gewalt durch stigmatisierende Einstellungen gefördert?

5. Verfestigt diese Praxis Stereotype wie z.B., dass von Menschen mit psychischer Erkrankung eine Gefahr ausgehe? Trägt sie zum Stigma bei?

6. Werden durch Unterbringung und weiterführende Beschränkungen die sozialen Beziehungen von Betroffenen verändert?

7. Welchen Stellenwert haben die Kontextfaktoren der psychiatrischen Behandlung oder die 
Einstellungen und Wertvorstellungen des behandelnden Personals auf die Praxis von Zwangsmaßnahmen?

8. Welchen Einfluss hat die Notwendigkeit auf Patienten Zwang und Gewalt ausüben zu müssen auf das Befinden, die Identität und die Rolle der damit betrauten Pflegepersonen und Ärzte?

9. Welche Auswirkungen haben die Gewaltanteile psychiatrischen Handelns auf das Image der Psychiatrie in der Gesellschaft?

Empirische Ergebnisse zu diesen Themen könnten zum Überdenken der gängigen Praxis, zu einer Reduktion von Zwang und zur Entwicklung von Behandlungsstandards beitragen [10].

Es wurde bislang kaum der Frage nachgegangen, wie Patienten, die in der Psychiatrie von Zwang oder Gewalt betroffen waren, diese erlebt haben. Die wenigen dazu vorhandenen Studien weisen darauf hin, dass das Erlebnis einer unfreiwilligen Hospitalisierung oder von weiterführenden Beschränkungen wie Fixierung oder Zwangsmedikation, häufig als ein traumatisierendes und stigmatisierendes Ereignis bewertet werden [11-15]. In einer eigenen Untersuchung konnten wir erfahren, dass Betroffene, trotz des durch Fixierung und Zwangsbehandlung zugefügten Traumas, sich konstruktiv mit dieser Thematik auseinandersetzten und uns auch Empfehlungen gaben, wie diese Praxis aus ihrer Sicht verhindert oder humaner gestaltet werden könnte.

Einblicke, wie Pfleger und Ärzte die Ausübung von Zwang und Gewalt erleben und verarbeiten, fehlen. In der Psychiatrie Tätige neigen dazu, auf diesen Bereich mit Abwehr, Schuldgefühlen oder auch unreflektierten Rechtfertigungen zu reagieren. Gewaltanwendung gegenüber Schwachen, Kranken oder Behinderten steht im Widerspruch zu den ethischen Grundhaltungen und sie passt nicht zum Selbstbild helfender Berufe. Diese Ablehnung kann dazu führen, dass die Gewaltanteile psychiatrischer Arbeit tabuisiert und in der Folge intransparent und schwer kontrollierbar werden. Dadurch entsteht auch Unsicherheit, ob sich die Frage nach der Angemessenheit der Zwangsausübung gegenüber Patienten eindeutig beantworten lässt; sie kann dann entweder als notwendiges Hilfsmittel zur Abwehr einer größeren Gefahr, oder auch als Ausdruck von Machtmissbrauch und Willkür interpretiert werden. Die Diskussion zu dieser Thematik wird häufig ideologisch geführt. Dies kann auch zur Spaltung innerhalb der Psychiatrie führen, wobei zwischen „guter Therapie“ und „böser Gewalt" unterschieden wird [16].

Es ist eine Konfrontation mit dem Spannungsfeld erforderlich, wie die Psychiatrie ihrer therapeutischen Rolle und der Funktion der gesellschaftlichen Kontrolle angemessen gerecht werden kann. Die Ausübung der Ordnungsfunktion ist nur dann gerechtfertigt, wenn durch sie eine psychisch kranke Person vor einer akuten und ernsthaften Gefährdung für Leib und Leben, die als Folge der Erkrankung besteht, geschützt werden muss. Was den Behandlungsauftrag betrifft, sollte nach Hartmann Hinterhuber ein Kompromiss gesucht werden, da der Verzicht von Zwang in der Behandlung u.U. zu einer Unterlassung von lebensnotwendigen Hilfestellungen führen kann: „Eine patientenzentrierte Ethik in der Psychiatrie muss stets um einen Ausgleich zwischen dem traditionellen paternalistischen Patientenwohl und dem radikalen Selbstbestimmungsrecht des Patienten bemüht sein."Was den legitimierten Auftrag zur Freiheitsbeschränkung betrifft, darf sich die Psychiatrie nicht von gesellschaftspolitischen Strömungen beeinflussen lassen $[17,18]$. Daher muss das enge Korsett bestehend aus gesetzlichen Regelungen und Legitimationsverpflichtung erhalten bleiben. Gerade weil die Auseinandersetzung mit dieser Thematik unter einem hohen Tabuisierungsdruck steht, ist es, da die gewaltfreie Psychiatrie heute noch eine Fiktion darstellt [16], erforderlich, dass dieser Teil psychiatrischen Handelns immer wieder kritisch durchleuchtet wird. Dazu äußerte Daniel Hell 1989: „Zwangsmaßnahmen sind für die stationäre Psychiatrie zwar nicht charakteristisch, aber sie sind für die Betroffenen - die „Opfer“ wie die „Täter“ - so belastend, dass sie immer wieder eine vertiefte Auseinandersetzung über Sinn und Widersinn nötig machen." In den Diskurs sollten alle Beteiligten - auch die Patienten - eingebunden werden. Er könnte ein Schutz vor unreflektierter "Gewaltroutine“ oder unangemessener Gewalt gegenüber psychisch erkrankten Menschen sein. Diese Diskussion kann auch dazu beitragen, dass das Stigma psychisch Kranker verringert und das Image der Psychiatrie in der Öffentlichkeit verbessert wird.

\section{Literatur}

1 Jäger $M$, Rössler $W$. Informeller Zwang zur Verbesserung der Behandlungsbereitschaft psychiatrischer Patienten. Neuropsychiatr 2009; 23: 206-215

2 Schott H, Tölle R. Geschichte der Psychiatrie. München: C. H. Beck Verlag; 2005

3 Dressing H, Salize H. Compulsory admission of mentally ill patients in European Union Member States. Soc Psychiatry Psychiatr Epidemiol 2004; 39: 797-803

4 Steinert T, Lepping P, Bernhardsgrütter $R$ et al. Incidence of seclusion and restraint in psychiatric hospitals: a literature review and survey of international trends. Soc Psychiatry Psychiatr Epidemiol 2010; 45: 889-897

5 Martin V, Bernhardsgrütter R, Göbel R et al. Ein Vergleich von Schweizer und deutschen Kliniken in Bezug auf die Anwendung von Fixierung und Isolierung. Psychiat Prax 2007; 34 (Suppl. 2): S212-217

6 Salize HJ, Dressing H. Coercion, involuntary treatment and quality of mental health care: is there any link? Curr Opin Psychiatry 2005; 18: 576-584

7 Europäische Kommission. Grünbuch - Die psychische Gesundheit der Bevölkerung verbessern. Brüssel: 2005 http://ec.europa.eu/health/ ph_determinants/life_style/mental/green_paper/mental_gp_de.pdf

8 Europarat/Ministerkomitee. Empfehlung Nr. Rec (2004) 10 an die Mitgliedsstaaten zum Schutz der Menschenrechte und der Würde von Personen mit psychischer Störung. Ministerkomitee; 896. Treffen; 2004

9 Kallert TW. Coercion in psychiatry. Curr Opin Psychiatry 2008; 21: 485-489

10 Steinert T, Bohnet $U$, Flammer $E$ et al. Effects of a training of power and balance on the use of mechanical restraint among in-patients with dementia. Psychiat Prax 2009; 36: 273-278

11 Frueh B, Knapp R, Cusack K et al. Patients' reports of traumatic or harmful experiences within the psychiatric setting. Psychiatr Serv 2005; 56: 1123-1133

12 Olofsson B, Jacobsson L. A plea for respect: involuntarily hospitalized psychiatric patients'narratives about being subjected to coercion. J Psychiatr Ment Health Nurs 2001; 8: 357-366

13 Längle G, Bayer W. Psychiatrische Zwangsbehandlung und die Sichtweise der Patienten. Psychiat Prax 2007; 34 (Suppl. 2): S203-207

14 Scheutz A, Amering M, Sibitz I. Zwangsmaßnahmen in der Psychiatrie: Auswirkungen auf das Leben von Betroffenen. Universitätsklinik für Psychiatrie, Medizinische Universität Wien; 2008

15 Längle G, Renner G, Günthner A et al. Psychiatric commitment: patients' perspectives. Med Law 2003; 22: 39-53

16 Wienberg G. Gewaltfreie Psychiatrie - eine Fiktion. In: Eink M, ed. Gewalttätige Psychiatrie - Ein Streitbuch. Bonn: Psychiatrie Verlag; 1997: $14-28$

17 Lauber C, Nordt C, Falcato L et al. Public attitude to compulsory admission of mentally ill people. Acta Psychiatr Scand 2002; 105: 385-389

18 Hinterhuber $H$, Lehofer $M$, Ofner $H$ et al. Verhaltenscodex für Psychiater. Neuropsychiatr 2009; 23: 263-266 